December 2006

\title{
The spectrum of massive excitations of $3 d$ 3-state Potts model and universality
}

\author{
R. Falcone*, R. Fiore*, M. Gravina* and A. Papa* \\ Dipartimento di Fisica, Università della Calabria, \\ and Istituto Nazionale di Fisica Nucleare, Gruppo collegato di Cosenza \\ I-87036 Arcavacata di Rende, Cosenza, Italy
}

\begin{abstract}
We consider the mass spectrum of the $3 d$ 3-state Potts model in the broken phase (a) near the second order Ising critical point in the temperature - magnetic field plane and (b) near the weakly first order transition point at zero magnetic field. In the case (a), we compare the mass spectrum with the prediction from universality of mass ratios in the $3 d$ Ising class; in the case (b), we determine a mass ratio to be compared with the corresponding one in the spectrum of screening masses of the $(3+1) d \mathrm{SU}(3)$ pure gauge theory at finite temperature in the deconfined phase near the transition. The agreement in the comparison in the case (a) would represent a non-trivial test of validity of the conjecture of spectrum universality. A positive answer to the comparison in the case (b) would suggest the possibility to extend this conjecture to weakly first order phase transitions.
\end{abstract}

* e-mail addresses: rfalcone,fiore,gravina,papa@cs.infn.it 


\section{Introduction}

Universality is a powerful concept since it establishes the common long-distance behavior of theories characterized by different microscopic interactions, but possessing the same underlying global symmetry. A remarkable example is provided by $(d+1)$-dimensional SU(N) pure gauge theories at finite temperature which undergo a confinement/deconfinement phase transition associated with the breaking of the center of the gauge group, $\mathrm{Z}(\mathrm{N})$, the order parameter being the Polyakov loop [1, 2]. When the transition is second order, the long-range critical behavior of $(d+1)$-dimensional $\mathrm{SU}(\mathrm{N})$ pure gauge theories at finite temperature is conjectured to be the same as the $d$-dimensional $\mathrm{Z}(\mathrm{N})$ spin model in the critical region near the order/disorder phase transition [3]. As a consequence, the gauge theory and the spin model are predicted to have the same critical indices, amplitude ratios and correlation functions at criticality. This prediction has been accurately verified in several cases - see, for instance, Refs. [4, 5, 6, 7, 8, 9, 10] and, for a review, Ref. [11].

A few years ago a study of the broken symmetry phase of the $3 d \mathrm{Z}(2)$ (Ising) class has brought compelling evidence that universality has a much wider reach than usually expected. In particular, it has been shown that the Ising model and the lattice regularized $\phi^{4}$ theory both exhibit a rich spectrum of massive excitations and that mass ratios coincide in the scaling region [12, 13]. This result is quite far from obvious: since only the lowest mass contributes to the free energy, there is no simple reason why higher masses in the spectrum should be universal. Later on, numerical evidence has been given that the same spectrum characterizes $(3+1) d \mathrm{SU}(2)$ gauge theory at finite temperature in the scaling region above the deconfinement temperature [14, 15]. In the deconfined phase of the $\mathrm{SU}(2)$ gauge theory the counterpart of the massive excitations in the broken phase of the $3 d$ Ising model are the so-called screening masses, i.e. the inverse exponential decay lengths of correlation functions between suitably defined operators, built from the Polyakov loops [14, 15].

It would be quite interesting to extend the test of the universality of the spectrum in two different directions: (1) by considering a theory with a global symmetry different from $\mathrm{Z}(2)$, which, however, presents in its phase diagram a second order critical point in the $3 d$ Ising class; (2) by verifying if and to what extent the universality of the spectrum holds also in the broken symmetry phase near a weak first order phase transition.

The $3 d$ 3-state Potts model with external magnetic field model provides a good testfield for both these investigations. Indeed, its phase diagram in the temperature - magnetic field $(T, h)$ plane (see Fig. 1) exhibits a line of first order phase transitions which starts at $(0,0)$, moves along the $T$-axis up to a transition temperature $T_{t}$ and then bends in the positive $h$-plane up to reaching a second order endpoint, which belongs to the Ising class [16]. The $3 d 3$-state Potts model near this critical endpoint should exhibit a mass spectrum in the Ising universality class. Instead, the mass spectrum of the $3 d$ 3-state Potts model at zero magnetic field, in the broken phase near $T_{t}$, i.e. for $T \lesssim T_{t}$ should reproduce the spectrum of screening masses of the finite temperature $(3+1) d \mathrm{SU}(3)$ pure gauge theory in the deconfined phase near criticality, if universality holds also in the case of weak first order phase transitions. 


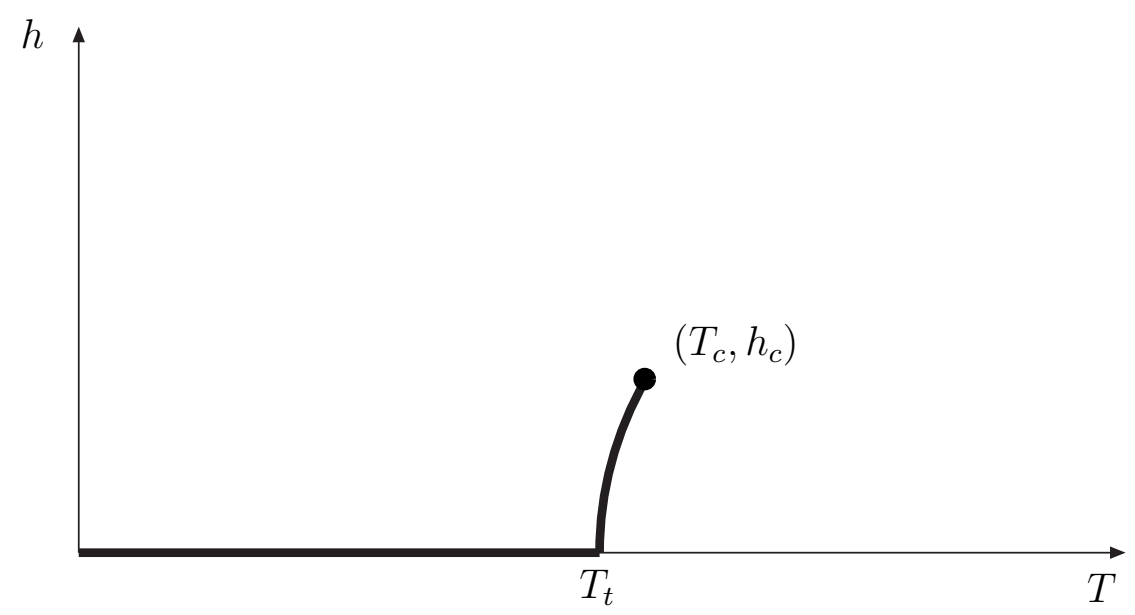

Figure 1: Qualitative phase diagram of the $3 d 3$-state Potts model: the solid line in bold is the line of first order phase transitions. $T_{t}$ is the order/disorder transition temperature at zero magnetic field, $\left(T_{c}, h_{c}\right)$ is the endpoint in the $3 d$ Ising class.

In this paper we determine the low-lying masses of the spectrum of the $3 d 3$-state Potts model in two different sectors of parity and orbital angular momentum, $0^{+}$and $2^{+}$, and in two different regions of the $(T, h)$ phase diagram, (a) near the critical Ising endpoint, (b) near the transition temperature $T_{t}$ at zero magnetic field. For the determinations in the region (a), the comparison between the resulting mass ratios and the corresponding ones in the $3 d$ Ising model [12] will provide a check of validity of the Ansatz of universality of the spectrum. The mass ratios determined in the region (b) are instead the quantities to be compared with the corresponding screening masses in the finite temperature $\mathrm{SU}(3)$ gauge theory in order to verify the validity of the Ansatz near a weakly first order transition point.

This paper is organized as follows: in Section 2 we briefly review the $3 d$ 3-state Potts model and describe its phase diagram on the temperature - magnetic field plane; in Section 3 we describe the method used to extract the massive excitations in the two regions of the phase diagram in which we are interested; in Section 4 we present our numerical results and in the last Section we draw our conclusions.

\section{The $3 d$ 3-state Potts model}

The 3- $d$ 3-state Potts model [17, 18] is a spin theory in which the fundamental degree of freedom, $s_{i}$, defined in the site $i$ of a 3 -dimensional lattice, is an element of the $\mathrm{Z}(3)$ group, i.e.

$$
s_{i}=e^{i \frac{2}{3} \pi \sigma_{i}}, \quad \sigma_{i}=\{0,1,2\} .
$$

The Hamiltonian of the model is

$$
H=-\frac{2}{3} \beta \sum_{\langle i j\rangle}\left(s_{i}^{\dagger} s_{j}+s_{j}^{\dagger} s_{i}\right)=-\beta \sum_{\langle i j\rangle} \delta_{\sigma_{i}, \sigma_{j}},
$$


up to an irrelevant constant. Here, $\beta$ is the coupling in units of the temperature and the sum is done over all the nearest-neighbor pairs of a cubic lattice with linear size $L$.

The Hamiltonian (2) is invariant under the $\mathrm{Z}(3)$ transformation

$$
s_{i} \longrightarrow s_{i}^{\prime}=e^{i \frac{2 \pi}{3} \sigma} s_{i},
$$

where $\sigma$ is fixed at any of the values $\{0,1,2\}$. It is well known that this system undergoes a weakly first order phase transition [19], associated with the spontaneous breaking of the $\mathrm{Z}(3)$ symmetry. The order parameter of this transition is the magnetization,

$$
\langle S\rangle=\left\langle\frac{1}{L^{3}} \sum_{i} s_{i}\right\rangle,
$$

which, in the thermodynamic limit, is zero above the transition temperature $T_{t}$ (or below the transition coupling $\beta_{t}$ ) and takes a non-zero value below $T_{t}$ (or above $\beta_{t}$ ).

In presence of an external magnetic field it is convenient to work with an Hamiltonian written in terms of the $\sigma_{i}$ degrees of freedom, instead of the $s_{i}$ ones. For a uniform magnetic field along the direction $\sigma_{h}$ with strength $h$ in units of the temperature, the Hamiltonian is

$$
H=-\beta \sum_{\langle i j\rangle} \delta_{\sigma_{i}, \sigma_{j}}-h \sum_{i} \delta_{\sigma_{i}, \sigma_{h}} \equiv-\beta E-h M
$$

where $E$ is the internal energy and $M$ is the magnetization. The magnetic field breaks explicitly the Z(3) symmetry. However, first order transitions still occur for values of the magnetic field strength $h$ below a critical value $h_{c}$, the transition temperature increasing with increasing $h$. The line of first order phase transitions ends in a second order critical point $P_{c}=\left(T_{c}, h_{c}\right)$ (see Fig. 1), belonging to the $3 d$ Ising class [16]. The Hamiltonian in the scaling region of the critical point $P_{c}$ can be written as

$$
H=-\tau \tilde{E}-\xi \tilde{M}
$$

where $\tilde{E}$ and $\tilde{M}$ are the Ising-like energy and magnetization operators and $\tau$ and $\xi$ the corresponding temperature-like and symmetry-breaking-like parameters. This means that $\langle\tilde{M}\rangle$ is the new order parameter. Close enough to $P_{c}$, the following relations hold,

$$
\tilde{M}=M+s E, \quad \tilde{E}=E+r M,
$$

where the mixing parameters $(r, s)$ have been determined numerically for several lattice sizes $L$ in Ref. [16]. The $\tau$-direction identifies the first order line (see Fig. 22). In Ref. [16] the location of the critical point $P_{c}$ has been accurately determined: $P_{c}=$ $\left(\beta_{c}, h_{c}\right)=(0.54938(2), 0.000775(10))$ or, equivalently, $P_{c}=\left(\tau_{c}, \xi_{c}\right)=(0.37182(2), 0.25733(2))$.

\section{Massive excitations and Universality}

Among the quantities relevant in the description of a phase transition there is the correlation function of the local order parameter: in the case of the $3 d$-state Potts model 


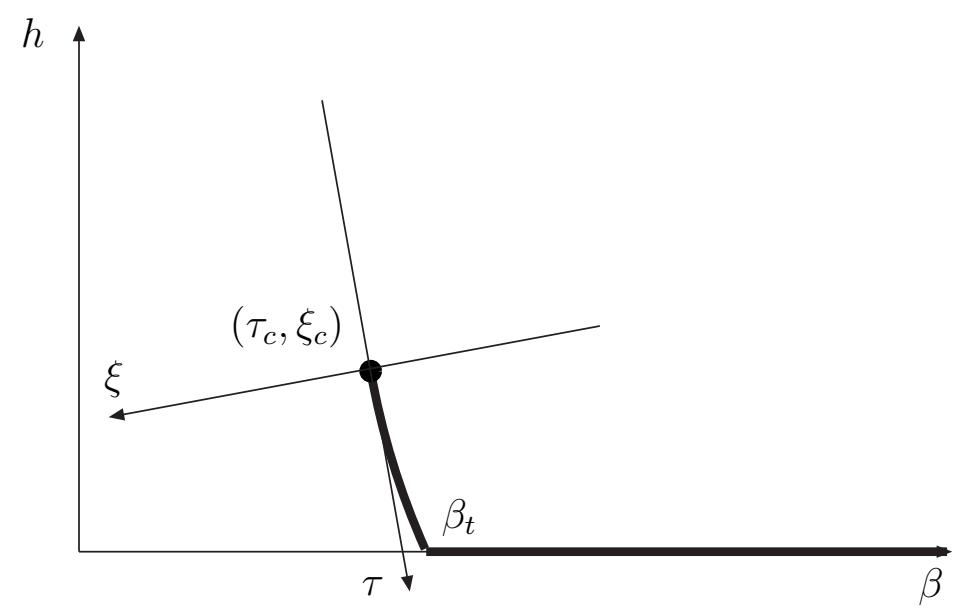

Figure 2: Qualitative phase diagram of the $3 d$ 3-state Potts model in the $(\beta, h)$-plane: $\xi$ and $\tau$ are the symmetry-breaking and the temperature parameters of the Ising theory (6).

this is just the local spin $s_{i}$. The point-point correlation function is defined as

$$
\Gamma_{i_{0}}(r)=\left\langle s_{i}^{\dagger} s_{i_{0}}\right\rangle-\left\langle s_{i}^{\dagger}\right\rangle\left\langle s_{i_{0}}\right\rangle,
$$

where $i$ and $i_{0}$ are the indices of the sites and $r$ is the distance between them. The large- $r$ behavior of the point-point correlation function is determined by the correlation length of the theory, $\xi_{0}$, or, equivalently, by its inverse, the fundamental mass. In order to extract the fundamental mass it is convenient to define the wall-wall correlation function, since numerical data in this case can be directly compared with exponentials in $r$, without any power prefactor. The connected wall-wall correlator in the $x$-direction is defined as

$$
G(x)=\frac{1}{L} \sum_{x_{0}}\left\langle w\left(x_{0}+x\right)^{\dagger} w\left(x_{0}\right)\right\rangle-\left\langle w\left(x_{0}+x\right)^{\dagger}\right\rangle\left\langle w\left(x_{0}\right)\right\rangle,
$$

where

$$
w(x)=\frac{1}{L^{2}} \sum_{y, z} s_{\{x, y, z\}}
$$

represents the spin average over the "wall" at the coordinate $x$.

The general behavior for the function $G(x)$ is

$$
G(x)=\sum_{n} a_{n} e^{-m_{n} x},
$$

where $m_{0}$ is the fundamental mass, while $m_{1}, m_{2}, \ldots$ are higher masses with the same angular momentum and parity $\left(0^{+}\right)$quantum numbers of the fundamental mass. Mass excitations in channels different from $0^{+}$can be determined by a suitable redefinition of the wall average (10). The fundamental mass in a definite channel can be extracted from wall-wall correlators by looking for a plateau of the effective mass,

$$
m_{\mathrm{eff}}(x)=\ln \frac{G(x)}{G(x-1)},
$$


at large distances. Excited masses in the given channel can be found by the variational method [20, 21], which consists in defining several wall-averaged operators with the given quantum numbers and building the matrix of cross-correlations between them. The eigenvalues of this matrix are single exponentials of masses in the given channel. The possibility to determine masses above the fundamental one in a given channel relies on the ability to define operators which have a large overlap with the excited states. Usually, these operators are defined in order to probe different length scales. In the present work we considered only the $0^{+}$and the $2^{+}$channels; the local variables to be wall-averaged as in (10) have been defined in the following way:

$$
\begin{aligned}
& s_{\{x, y, z\}}^{0^{+}}(n)=s_{\{x, y, z\}}\left(s_{\{x, y+n, z\}}+s_{\{x, y, z+n\}}\right), \\
& s_{\{x, y, z\}}^{2^{+}}(n)=s_{\{x, y, z\}}\left(s_{\{x, y+n, z\}}-s_{\{x, y, z+n\}}\right) .
\end{aligned}
$$

Typically, we have used about ten operators in each channel, with the largest $n$ almost reaching $L$. We can anticipate that we have not been able to extract masses higher than the fundamental one in any of the two channels. However, the use of the variational method has improved considerably the evaluation of the fundamental $2^{+}$mass. Needless to say that the determination of the fundamental mass in the $0^{+}$channel by the definition (13) turned out to be in perfect agreement with that from (9).

A few years ago it has been proposed [12] that ratios between massive excitations in the broken phase are universal quantities, i.e. mass ratios must be equal in theories where the same mechanism of symmetry breaking at the transition is active. One of the motivations of the present work is to check whether this statement holds also for the broken phase of the Ising theory (6) near the critical endpoint $P_{c}$ of the $3 d 3$-state Potts model. This would provide us with a non-trivial check of validity of the conjecture of universal mass spectrum.

For the $3 d$ Ising universality class, a definite prediction exists for the ratio between the fundamental $0^{+}$and $2^{+}$masses, $m_{2^{+}} / m_{0^{+}}[12$. We can estimate the same ratio near the Ising endpoint of the $3 d$ 3-state Potts model and verify if it is consistent with this prediction. The procedure to determine the fundamental masses in the two channels of interest is the same outlined above, with the only difference that we need to use the correct local order parameter to build correlators. We have defined this local variable, $\tilde{m}_{i}$, in such a way that it reconstructs the global magnetization operator $\tilde{M}$ after summation over the whole lattice:

$$
\tilde{m}_{i}=\delta_{\sigma_{i} \sigma_{h}}+\frac{s}{2} \sum_{\hat{\mu}} \delta_{\sigma_{i} \sigma_{i+\hat{\mu}}} .
$$

Indeed, it is easy to see that $\tilde{M}_{i}=\sum_{i} \tilde{m}_{i}$.

The second aim of the present work is to study mass ratios also in the broken phase of the $3 d$ 3-state Potts model at zero magnetic field near the transition point. It is known that this transition is weakly first order, a signature of this being the fact that the correlation length $\xi$, though finite at the transition point, becomes much larger than the lattice spacing. This fact could open the door to universality effects, such as the abovementioned conjecture on mass ratios. If this claim is true, an important consequence 
would be that mass ratios in the broken phase near criticality of the $3 d$ 3-state Potts model at zero magnetic field should reproduce the corresponding ratios in the phase of broken $\mathrm{Z}(3)$ symmetry of the $(3+1) d \mathrm{SU}(3)$ pure gauge theory at finite temperature. This is the deconfined phase of the $\mathrm{SU}(3)$ gauge theory and the counterparts of the massive excitations of the spin model are the so-called screening masses, i.e. the inverse decay lengths of the Yukawa-like interaction potential between static color sources.

As a probe for the check of consistency of this scenario, we can consider again the mass ratio $m_{2^{+}} / m_{0^{+}}$. If it will turn out that its value near the transition coupling $\beta_{t}$ in the broken phase of the $3 d 3$-state Potts model is compatible with the corresponding ratio of screening masses in the deconfined phase near transition of $(3+1) d \mathrm{SU}(3)$ at finite temperature, this could be taken as an indication that the conjecture of universal spectrum can be extended also to the case of weakly first order transition.

Summarizing, we have to calculate the $m_{2^{+}} / m_{0^{+}}$ratio in two regions of interest in the temperature - magnetic field phase diagram: (a) in the broken phase near the critical Ising endpoint, (b) in the broken phase near the transition point of the model in absence of magnetic field. In the case (a), we have to compare the result with the predicted value from universality in the $3 d$ Ising class, given in Ref. [12]; in the case (b), our determination is to be compared with the corresponding one in the $\mathrm{SU}(3)$ pure gauge theory [22].

\section{Numerical results}

We have performed numerical Monte Carlo simulations of the $3 d$ 3-state Potts model using a cluster algorithm [23, 24] to reduce the autocorrelation effects. In order to minimize the finite volume effects, we have imposed periodic boundary conditions. Data analysis has been done by the jackknife method applied to bins of different lengths.

For the simulations near the critical endpoint $P_{c}$ (region (a)) we used lattices with size $L=70$; near the transition coupling $\beta_{t}$ at zero magnetic field (region (b)) we chose instead $L=48$. In both cases we have seen tunneling between degenerate minima near the transition point. This finite volume effect can spoil mass measurements in the scaling region and must be treated carefully. Depending on the order of the transition, tunneling effects show up differently and must be removed accordingly.

\subsection{Region (a): critical endpoint}

We have performed simulations on $70^{3}$ lattices for which the mixing parameters appearing in (17) turn to be $s(L=70)=-0.689(8)$ and $r(L=70)=0.690(3)$ [16].

First of all we have considered the distribution of the order parameter $\tilde{M}$ in the broken phase of the Ising theory (6) near $P_{c}$. Fig. 3 shows the structure, typical for a secondorder phase transition, with two peaks corresponding to the two degenerate minima in the broken phase which separate while moving away from $P_{c}$ along the first order Ising critical line. This double-peak structure is the signal of tunneling. The last plot in Fig. 3, obtained for Ising couplings $\xi=\xi_{c}$ and $\tau=0.37248$, shows two almost completely 
separated peaks. We have decided to choose this as our working point and performed here simulations with statistics $200 \mathrm{k}$.

We have removed here tunneling effects by the brute force method of analyzing separately data belonging to each peak. In Figs. 4 and 5 we show the behavior of the effective masses in the $0^{+}$and in the $2^{+}$channels at $\xi=\xi_{c}$ and $\tau=0.37248$, as functions of the separation between walls, for the configurations in the "right-peak". Similar plots have been obtained for the configurations in the "left-peak". In each case, the plateau mass is taken as the effective mass (with its error) belonging to the plateau and having the minimal uncertainty. We define plateau the largest set of consecutive data points, consistent with each other within $1 \sigma$. This procedure is more conservative than identifying the plateau mass and its error as the results of a fit with a constant on the effective masses $m_{\mathrm{eff}}(x)$, for large enough $x$. We have found

"right-peak" (statistics 115k)

$$
a m_{0^{+}}=0.0725(63), \quad a m_{2^{+}}=0.1981(87), \quad \frac{m_{2^{+}}}{m_{0^{+}}}=2.73(36) ;
$$

"left-peak" (statistics 85k)

$$
a m_{0^{+}}=0.0714(40), \quad a m_{2^{+}}=0.1959(80), \quad \frac{m_{2^{+}}}{m_{0^{+}}}=2.74(27) .
$$

The uncertainty on the mass ratios has been determined by usual propagation of errors. The two determinations of the masses and, therefore, of the mass ratios are consistent, as expected. Moreover, they are compatible with the value of the $3 d$ Ising class [12], $m_{2^{+}} / m_{0^{+}}=2.59(4)$.

\subsection{Region (b): transition point at zero magnetic field}

We have performed simulations on $48^{3}$ lattices for several values of the coupling $\beta$ in the broken phase of the $3 d$ 3-state Potts model at zero magnetic field. A summary of the Monte Carlo simulations is presented in Table 1.

Close enough to $\beta_{t}(L=48)=0.550538$, determined in Ref. [19], the scatter plot of the complex parameter $S$ shows the coexistence of the symmetric phase (points around $(0,0)$ in the $\operatorname{Im} S$ - Re $S$ plane in Fig. 6) and of the broken phase (points around the three roots of the identity in Fig. 6). Notice that the peaks in the distribution of points in Fig. 6 are well separated, as it must be for first order dynamics. In the thermodynamic limit this would occur only at the transition point; at finite volume, tunneling between broken minima and the symmetric phase occurs in a small region around the transition. The amplitude of this region decreases with the volume. For $L=48$ it is of the order of $10^{-4}$ in $\beta[19$.

Moving away from $\beta_{t}$ the symmetric phase becomes less and less important, up to disappearing. For $\beta=0.5508$ there are only the three broken minima, as shown in Fig. 7(top). For larger $\beta$ values the tunneling between broken minima survives. However, the three 
peaks are totally separated and it is therefore possible to "rotate" unambiguously all of them to the real sector (see Fig. 7 (bottom)). Working only in one sector allows us to optimize statistics. With this approach we have calculated the fundamental masses in the $0^{+}$and $2^{+}$channels for several $\beta$ values, up to $0.60\left(\left(T_{t}-T\right) / T_{t} \sim 0.08\right)$. In Figs. 8 and 9 we show the behavior of the effective masses in the $0^{+}$and in the $2^{+}$channel at $\beta=0.554$, as functions of the separation between walls. Similar plots have been obtained for the other $\beta$ values. The plateau mass values have been determined as described in the previous Subsection. In Table 1 we present our results, whereas in Fig. 10 we have plotted the behavior of $m_{0^{+}}$and $m_{2^{+}}$versus $\beta$.

Table 1: Fundamental masses in lattice units in the $0^{+}$and $2^{+}$channels and their ratio for $\beta$ values in the broken phase near the weakly first order transition at $L=48$. The statistics of each simulation is also given.

\begin{tabular}{|l|l|c|c|c|}
\hline$\beta$ & $m_{0^{+}}$ & $m_{2^{+}}$ & $m_{2^{+}} / m_{0^{+}}$ & statistics \\
\hline 0.5508 & $0.1556(36)$ & $0.381(17)$ & $2.45(17)$ & $300 \mathrm{k}$ \\
0.550875 & $0.1565(56)$ & $0.384(16)$ & $2.45(19)$ & $200 \mathrm{k}$ \\
0.551 & $0.1837(59)$ & $0.444(18)$ & $2.42(18)$ & $100 \mathrm{k}$ \\
0.552 & $0.2375(42)$ & $0.533(36)$ & $2.24(19)$ & $100 \mathrm{k}$ \\
0.553 & $0.2900(34)$ & $0.660(27)$ & $2.28(12)$ & $200 \mathrm{k}$ \\
0.554 & $0.3258(60)$ & $0.691(57)$ & $2.12(21)$ & $100 \mathrm{k}$ \\
0.555 & $0.3502(67)$ & $0.847(29)$ & $2.42(13)$ & $200 \mathrm{k}$ \\
0.556 & $0.3996(85)$ & $0.891(35)$ & $2.23(14)$ & $200 \mathrm{k}$ \\
0.56 & $0.4965(83)$ & $1.204(30)$ & $2.43(10)$ & $200 \mathrm{k}$ \\
0.562 & $0.537(11)$ & - & - & $200 \mathrm{k}$ \\
0.565 & $0.6324(85)$ & - & - & $200 \mathrm{k}$ \\
0.57 & $0.702(12)$ & - & - & $200 \mathrm{k}$ \\
0.575 & $0.8381(78)$ & - & - & $200 \mathrm{k}$ \\
0.58 & $0.9358(97)$ & - & - & $200 \mathrm{k}$ \\
0.60 & $1.170(20)$ & - & - & $200 \mathrm{k}$ \\
\hline
\end{tabular}

Since for first order transitions the critical exponent of the correlation length is $\nu=$ $1 / 3$ [25], the scaling law

$$
\left(\frac{\beta_{1}-\beta_{t}}{\beta_{2}-\beta_{t}}\right)^{\nu} \sim \frac{m_{0^{+}}\left(\beta_{1}\right)}{m_{0^{+}}\left(\beta_{2}\right)}
$$

must hold in the scaling region. Here $m_{0^{+}}\left(\beta_{1}\right)$ and $m_{0^{+}}\left(\beta_{2}\right)$ are the fundamental masses in the $0^{+}$channel at $\beta_{1}$ and $\beta_{2}$, respectively. Comparing the scaling function with the ratios determined from simulations, we can estimate how large is the scaling region. Fig. 11 shows that the scaling region extends at least up to $\beta=0.562\left(\left(T_{t}-T\right) / T_{t} \sim 0.02\right)$.

We have determined the ratio $m_{2^{+}} / m_{0^{+}}$for several $\beta$ values in the region $\left[\beta_{t}, 0.56\right]$; the results are presented in Table 1 and plotted in Fig. 12. This ratio remains practically constant in the considered region, thus suggesting that the correlation length $\xi_{2}$ associated 
to the channel $2^{+}\left(\xi_{2}=1 / m_{2^{+}}\right)$scales in the same way of the fundamental one $\left(\xi_{0}=\right.$ $\left.1 / m_{0^{+}}\right)$. We can take as our estimation of the mass ratio the value

$$
\frac{m_{2^{+}}}{m_{0^{+}}}=2.43(10)
$$

determined, as discussed above, by taking value and error of the point with the smallest error belonging to the plateau $(\beta=0.56$, see Fig. 12). A fit of the data with a constant gives for this ratio the value $2.353(49)$ with a $\chi^{2} /$ d.o.f. $=0.54$.

The result given in (18) is to be taken as a prediction for the corresponding ratio of the screening masses in the broken phase of the $(3+1) d \mathrm{SU}(3)$ pure gauge theory at finite temperature.

\section{Conclusions and outlook}

In this work we have studied massive excitations of the $3 d 3$-state Potts model near the Ising critical point on the temperature - magnetic field phase diagram and near the transition point at zero magnetic field.

We have found evidence that the mass ratio $m_{2+} / m_{0+}$ near the Ising critical point is compatible with the prediction from universality, thus supporting the conjecture of universal spectrum.

In the broken phase of the scaling region near the transition in absence of the external source, we have found $m_{2+} / m_{0+}=2.43(10)$. This result is to be compared with the corresponding ratio between screening masses of $(3+1) d \mathrm{SU}(3)$ pure gauge theory at finite temperature in the broken phase near the deconfinement temperature [22]. An agreement between these determinations would represent an indication that universality of the mass spectrum holds also for weakly first order transitions.

The present analysis could be extended with the numerical determination of higher masses in the $0^{+}$and $2^{+}$channels and with masses in other channels, in order to carry out a more systematic study of universality effects. Such an extension would be certainly well-motivated in case of a positive answer of the comparison with the $\mathrm{SU}(3)$ gauge theory.

\section{References}

[1] A.M. Polyakov, Phys. Lett. B 72 (1978) 477.

[2] L. Susskind, Phys. Rev. D 20 (1979) 2610.

[3] B. Svetitsky and L.G. Yaffe, Nucl. Phys. B 210 (1982) 423.

[4] F. Gliozzi and P. Provero, Phys. Rev. D 56 (1997) 1131.

[5] R. Fiore, F. Gliozzi and P. Provero, Phys. Rev. D 58 (1998) 114502.

[6] J. Engels and T. Scheideler, Nucl. Phys. B 539 (1999) 557. 
[7] S. Fortunato, F. Karsch, P. Petreczky and H. Satz, Nucl. Phys. (Proc. Suppl.) 94 (2001) 398.

[8] R. Fiore, A. Papa and P. Provero, Nucl. Phys. (Proc. Suppl.) 106 (2002) 486.

[9] R. Fiore, A. Papa and P. Provero, Phys. Rev. D 63 (2001) 117503.

[10] A. Papa and C. Vena, Int. J. Mod. Phys. A 19 (2004) 3209.

[11] A. Pelissetto and E. Vicari, Phys. Rept. 368 (2002) 549.

[12] M. Caselle, M. Hasenbusch and P. Provero, Nucl. Phys. B 556 (1999) 575.

[13] M. Caselle, M. Hasenbusch, P. Provero and K. Zarembo, Nucl. Phys. B 623 (2002) 474 .

[14] R. Fiore, A. Papa and P. Provero, Nucl. Phys. (Proc. Suppl.) 119 (2003) 490.

[15] R. Fiore, A. Papa and P. Provero, Phys. Rev. D 67 (2003) 114508.

[16] F. Karsch and S. Stickan, Phys. Lett. B 488 (2000) 319.

[17] H.W.J. Blöte and R.H. Swendsen, Phys. Rev. Lett. 43 (1979) 779.

[18] W. Janke and R. Villanova, Nucl. Phys. B 489 (1997) 679 and references therein.

[19] R.V. Gavai, F. Karsch and B. Petersson, Nucl. Phys. B 322 (1989) 738.

[20] A. S. Kronfeld, Nucl. Phys. (Proc. Suppl.) 17 (1990) 313.

[21] M. Lüscher and U. Wolff, Nucl. Phys. B 339 (1990) 222.

[22] R. Falcone, R. Fiore, M. Gravina and A. Papa, work in progress.

[23] R. H. Swendsen and J. S. Wang, Phys. Rev. Lett. 58 (1987) 86.

[24] P. W. Kasteleyn and C. M. Fortuin, J. Phys. Soc. Jpn. Suppl. 26 (1969) 11.

[25] M. E. Fisher and A. N. Berker, Phys. Rev. B 26 (1982) 2507. 

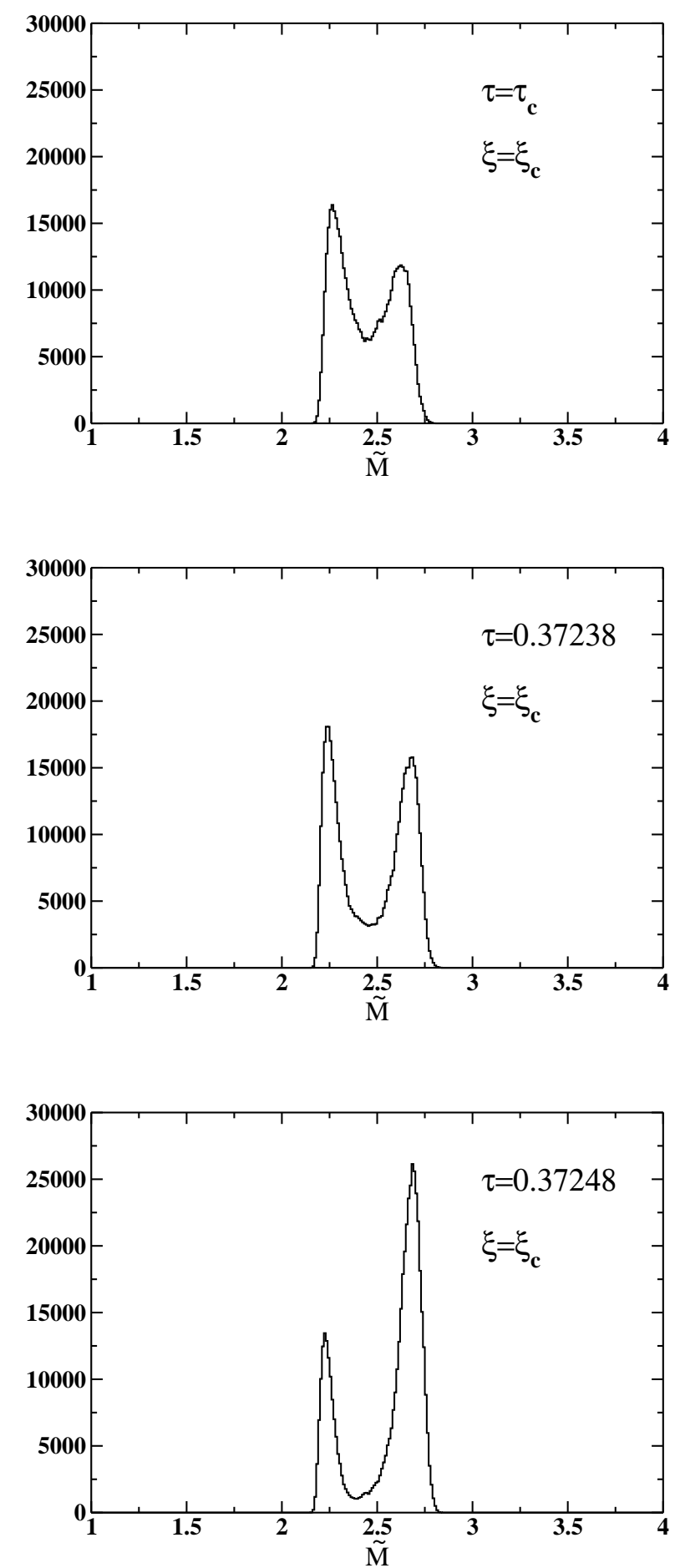

Figure 3: Distributions of the order parameter $\tilde{M}$ near $P_{c}$. The simulations have been done at the points $\left(\xi_{c}, \tau\right)$ with $\tau=0.37182 \equiv \tau_{c}, \tau=0.37238$ and $\tau=0.37248$. These points lie on the first order line starting from $P_{c}$. The statistics is $500 \mathrm{k}$ in all cases. 


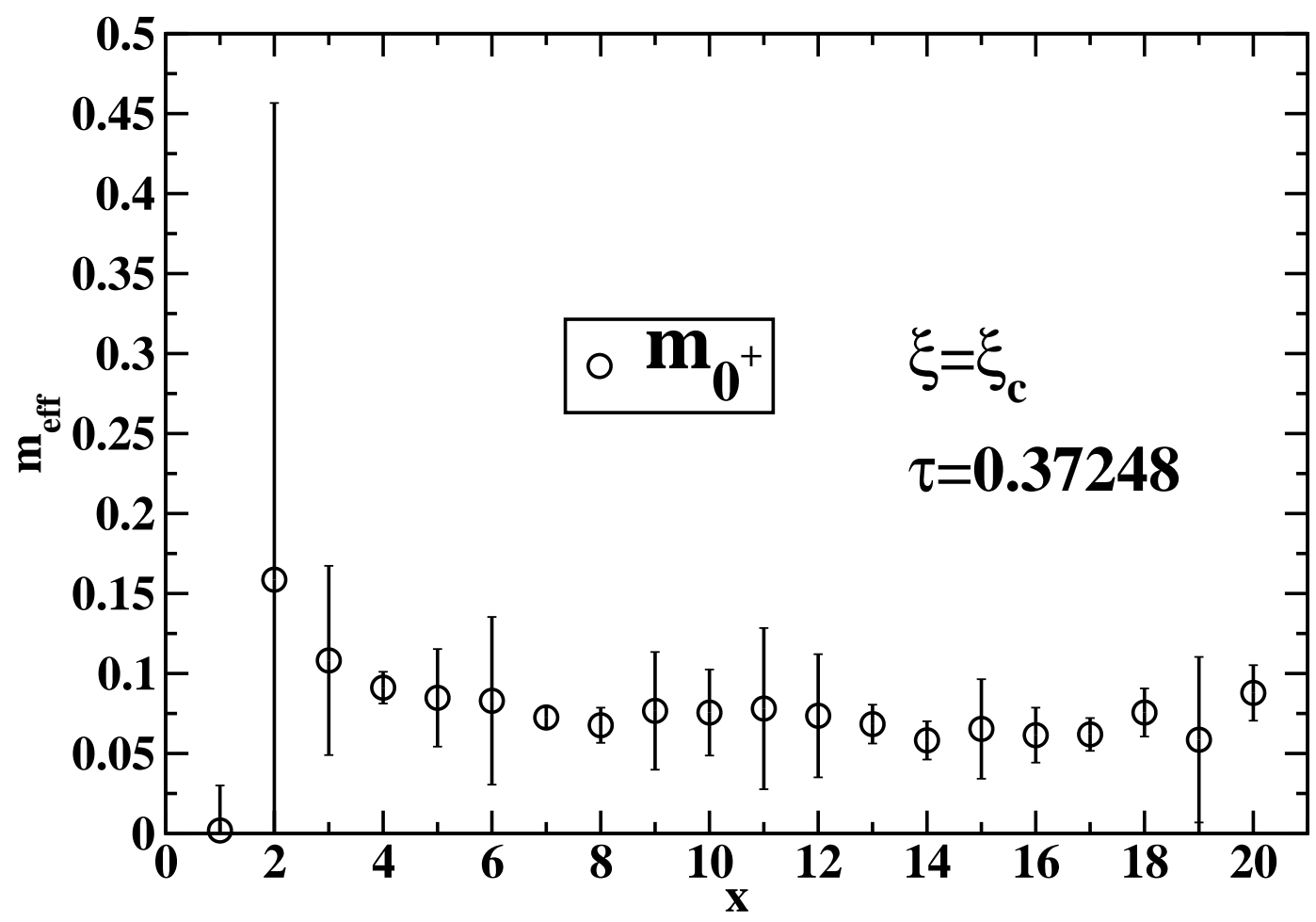

Figure 4: Effective mass in the $0^{+}$channel as a function of the separation between walls on the $(y, z)$ plane at $\xi=\xi_{c}$ and $\tau=0.37248$, determined from the configurations belonging to the "right-peak" in the thermal equilibrium ensemble. 


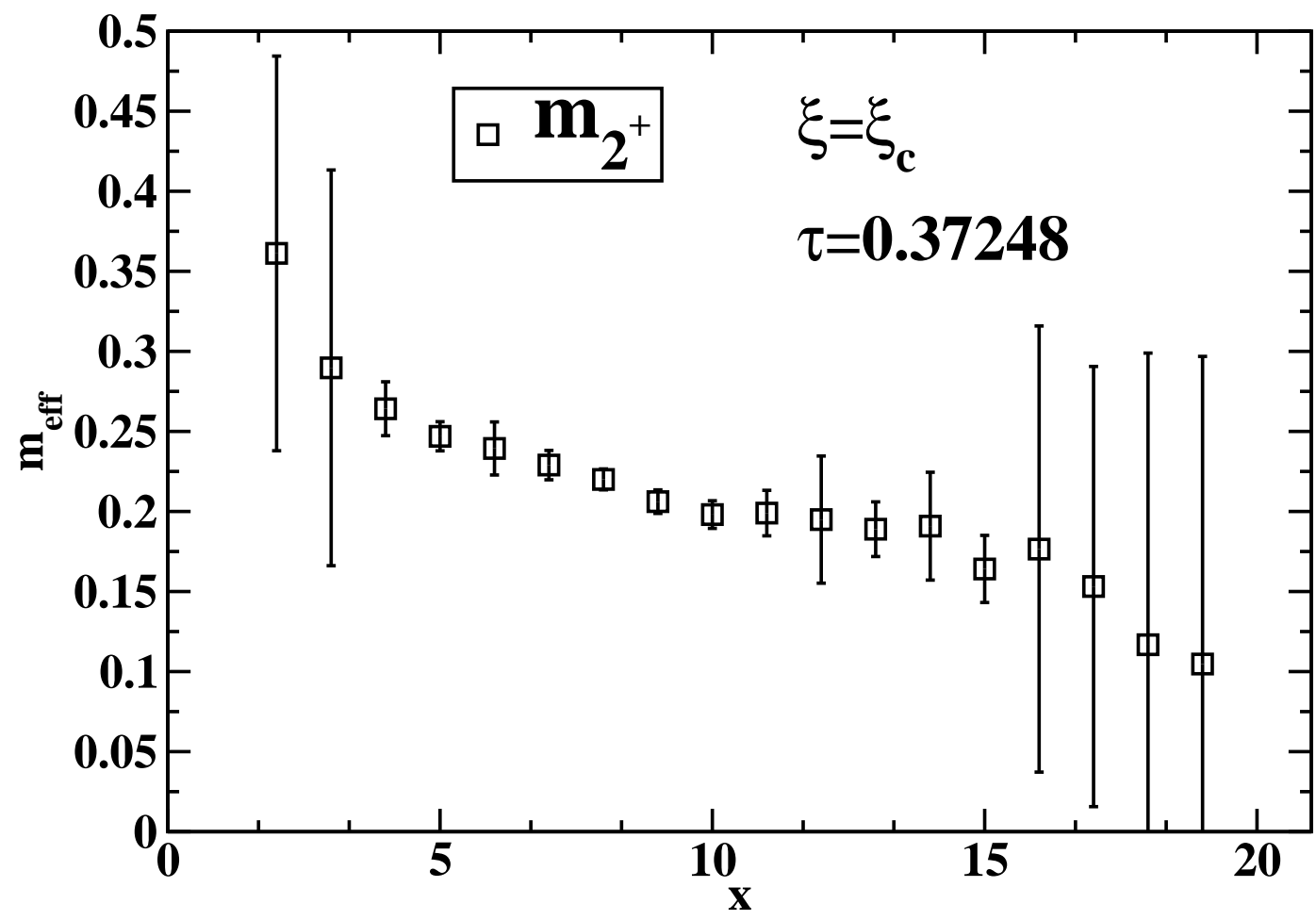

Figure 5: Effective mass in the $2^{+}$channel as a function of the separation between walls on the $(y, z)$ plane at $\xi=\xi_{c}$ and $\tau=0.37248$, determined from the configurations belonging to the "right-peak" in the thermal equilibrium ensemble.

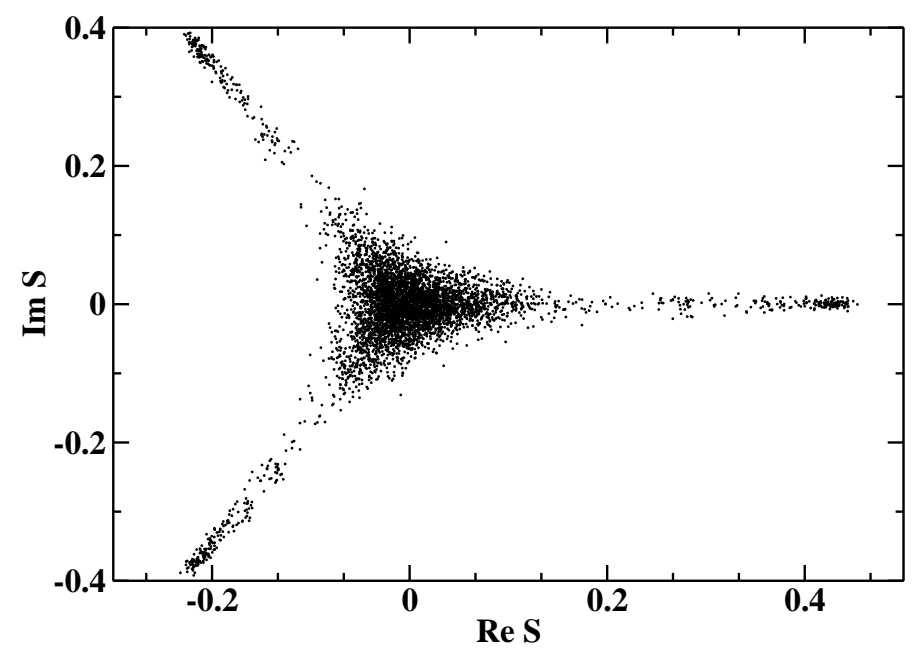

Figure 6: Scatter plot of the complex variable $S$ at the transition point $\beta_{t}=0.550538$ [19] at zero magnetic field. Both the symmetric and the broken phases are present. 

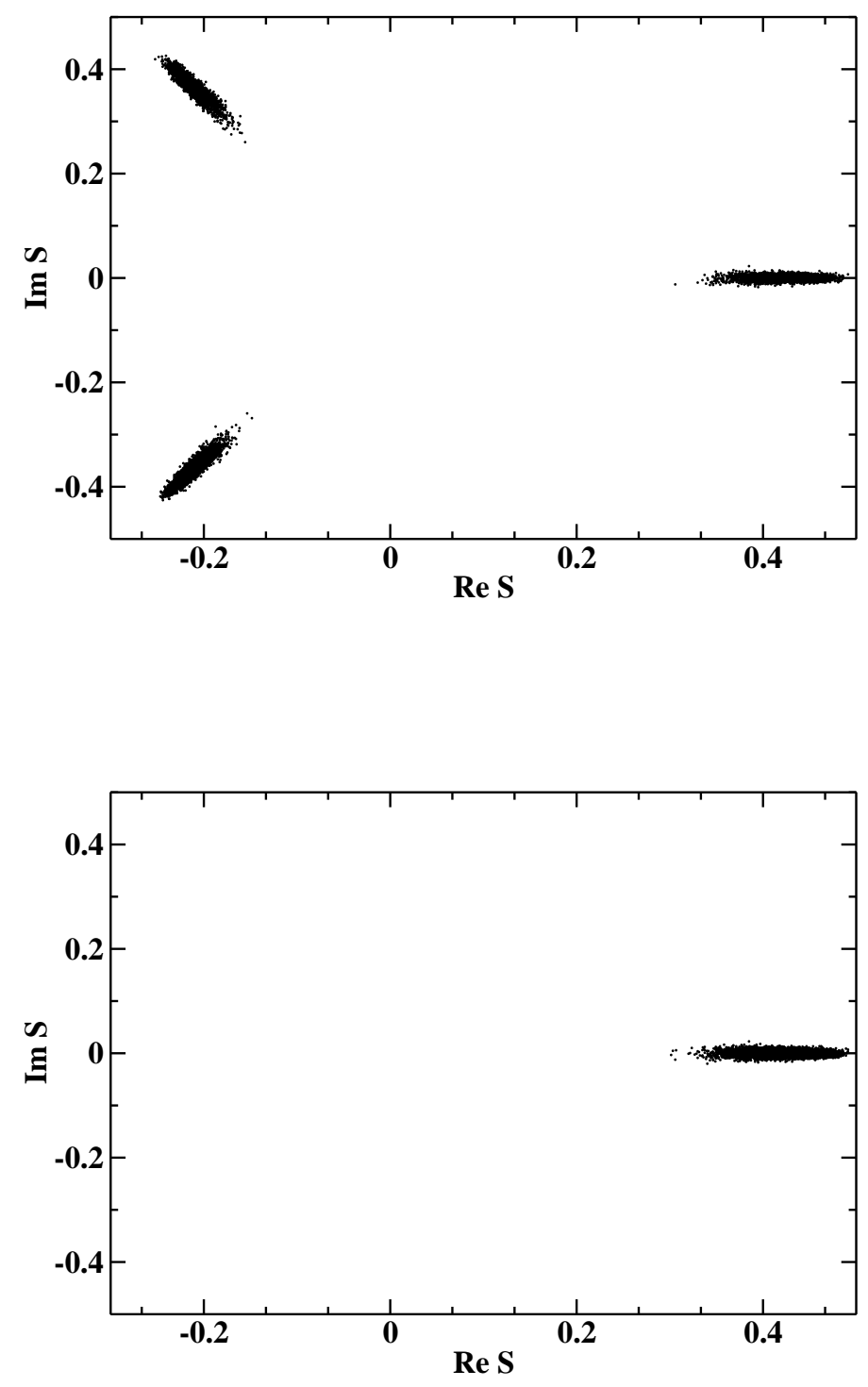

Figure 7: (Top) Typical scatter plot of the complex variable $S$ for $\beta$ larger than 0.5508 and zero magnetic field on a lattice $48^{3}$. There are no states in the symmetric phase, but tunneling survives between the three broken minima.

(Bottom) Same as (Top) with the tunneling between broken minima removed by the "rotation" to the real phase. 


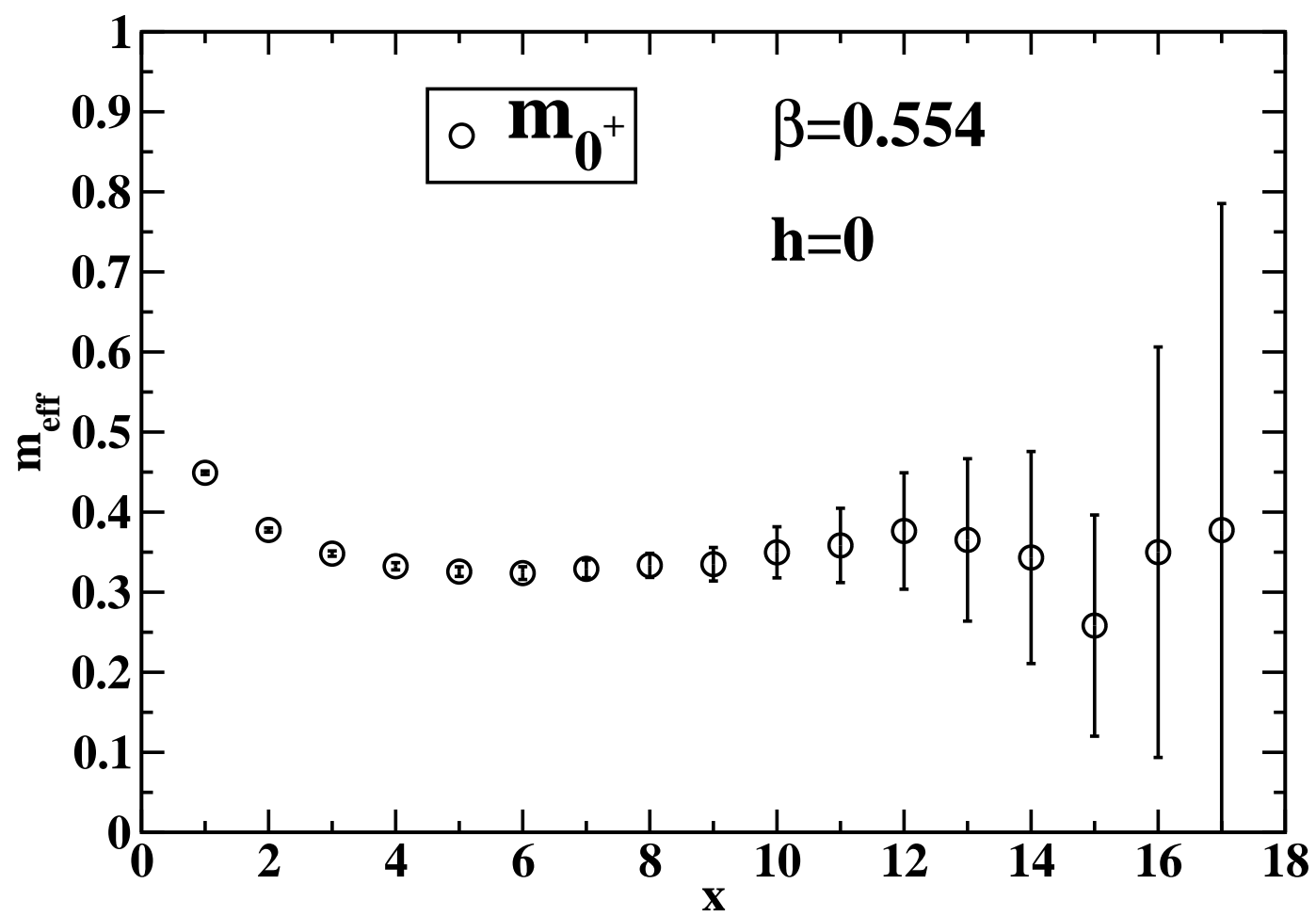

Figure 8: Effective mass in the $0^{+}$channel as a function of the separation between walls on the $(y, z)$ plane at $\beta=0.554$ and $h=0$. 


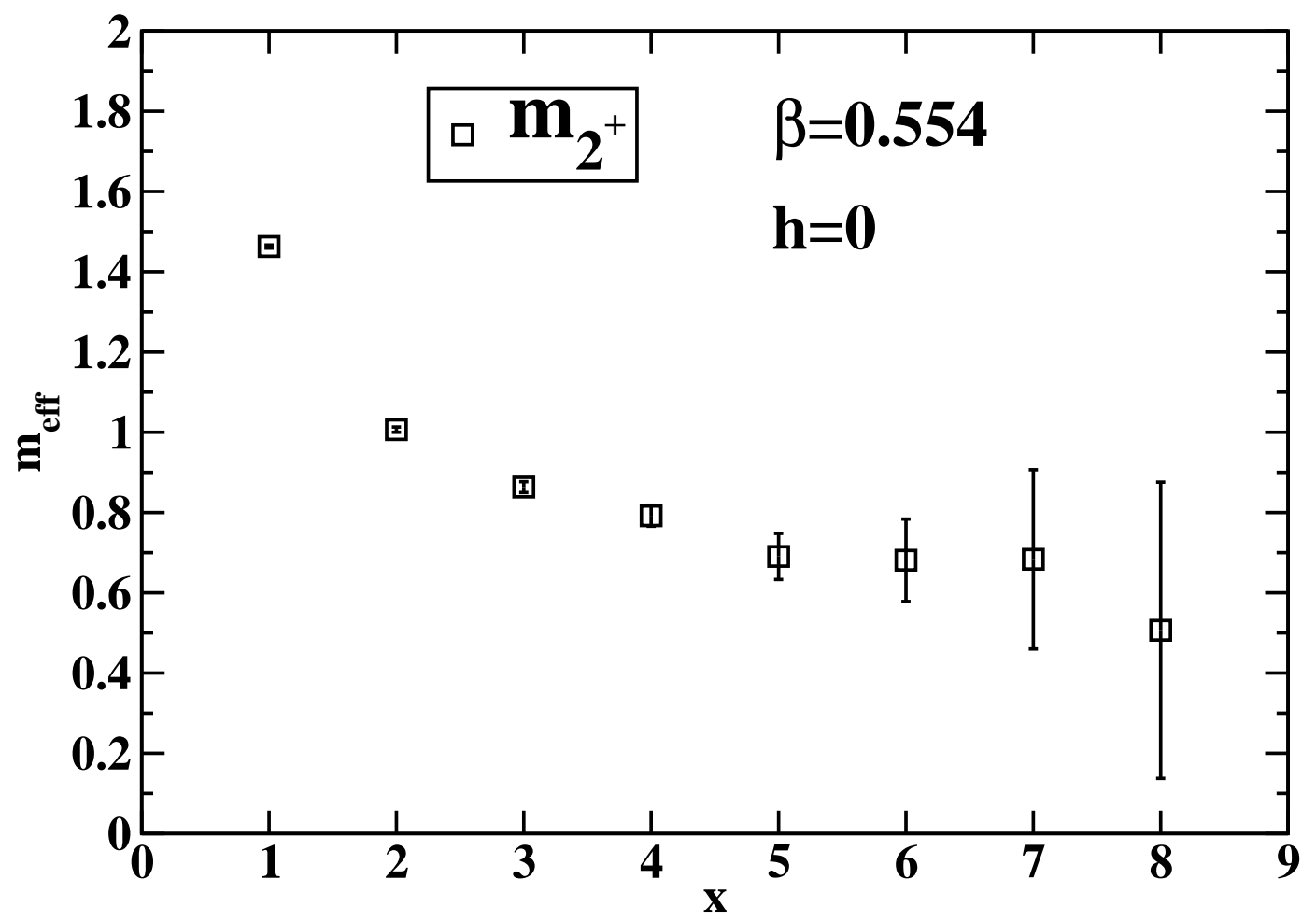

Figure 9: Effective mass in the $2^{+}$channel as a function of the separation between walls on the $(y, z)$ plane at $\beta=0.554$ and $h=0$. 


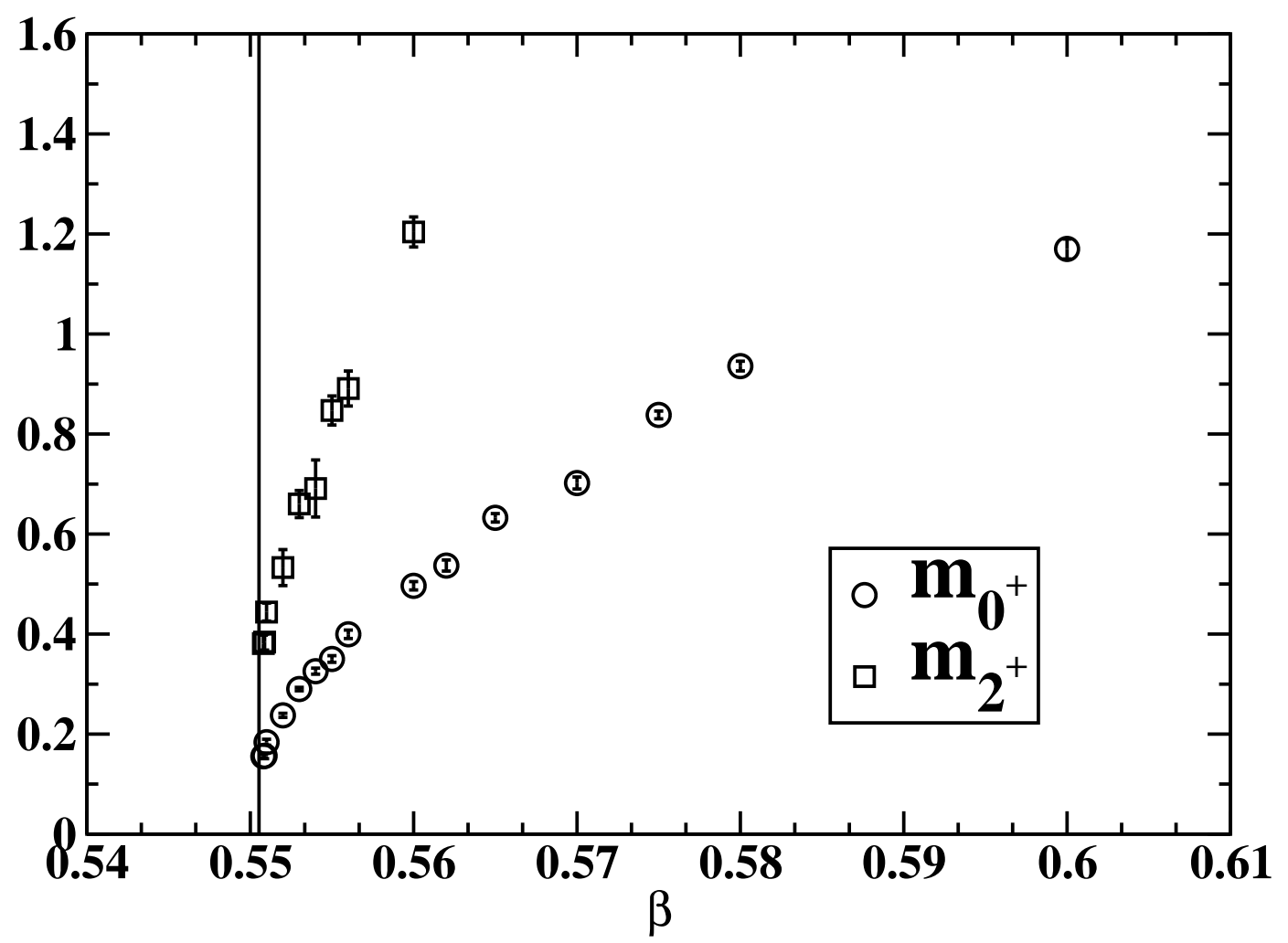

Figure 10: Fundamental masses in the $0^{+}$and $2^{+}$channels as functions of $\beta$, in the broken phase near $T_{t}$ (vertical line). 


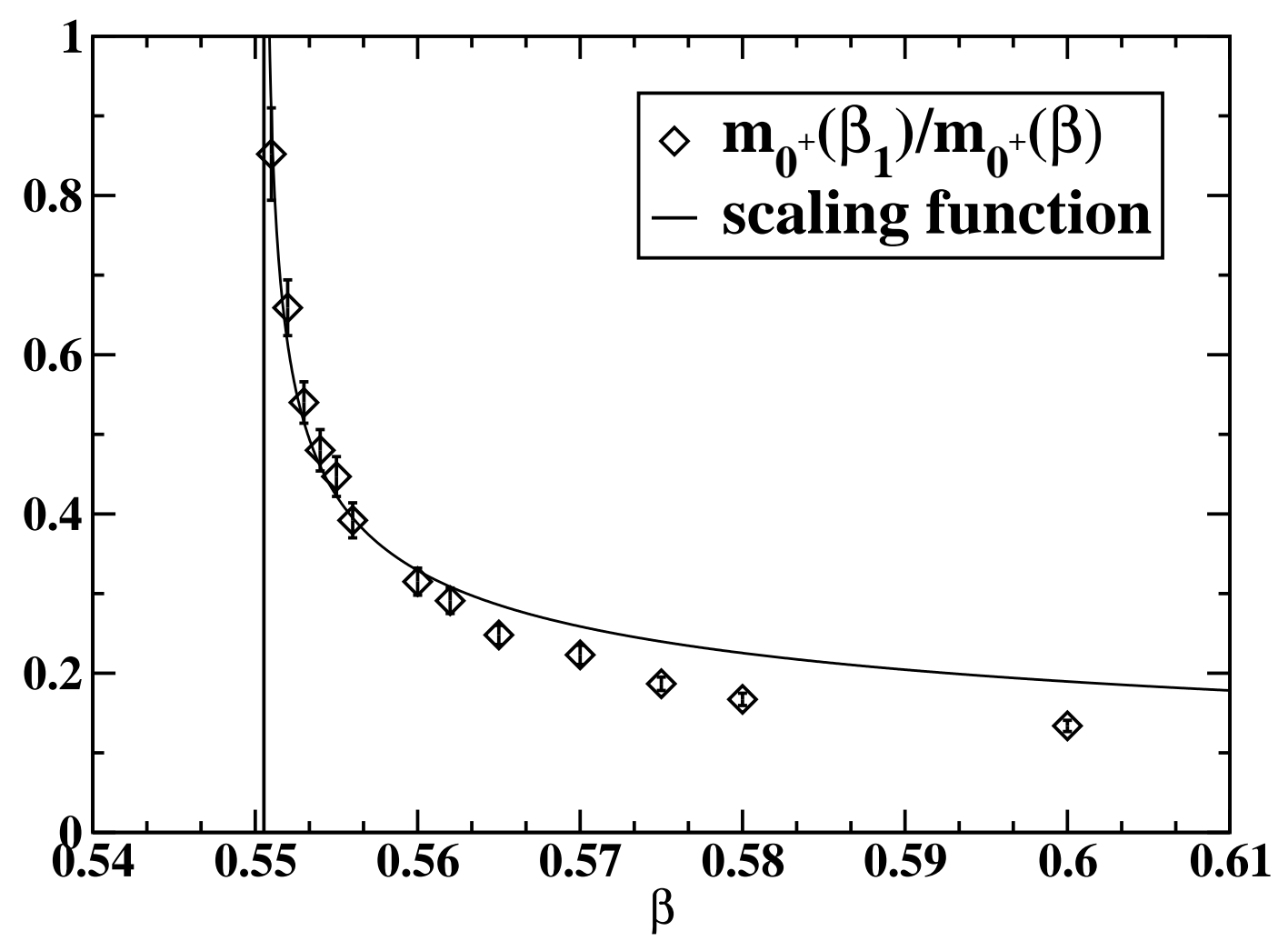

Figure 11: Comparison between the scaling function $\left[\left(\beta_{1}-\beta_{t}\right) /\left(\beta-\beta_{t}\right)\right]^{1 / 3}$ and the mass ratio $m_{0^{+}}\left(\beta_{1}\right) / m_{0^{+}}(\beta)$ for varying $\beta$, with $\beta_{1}=0.550875$. 


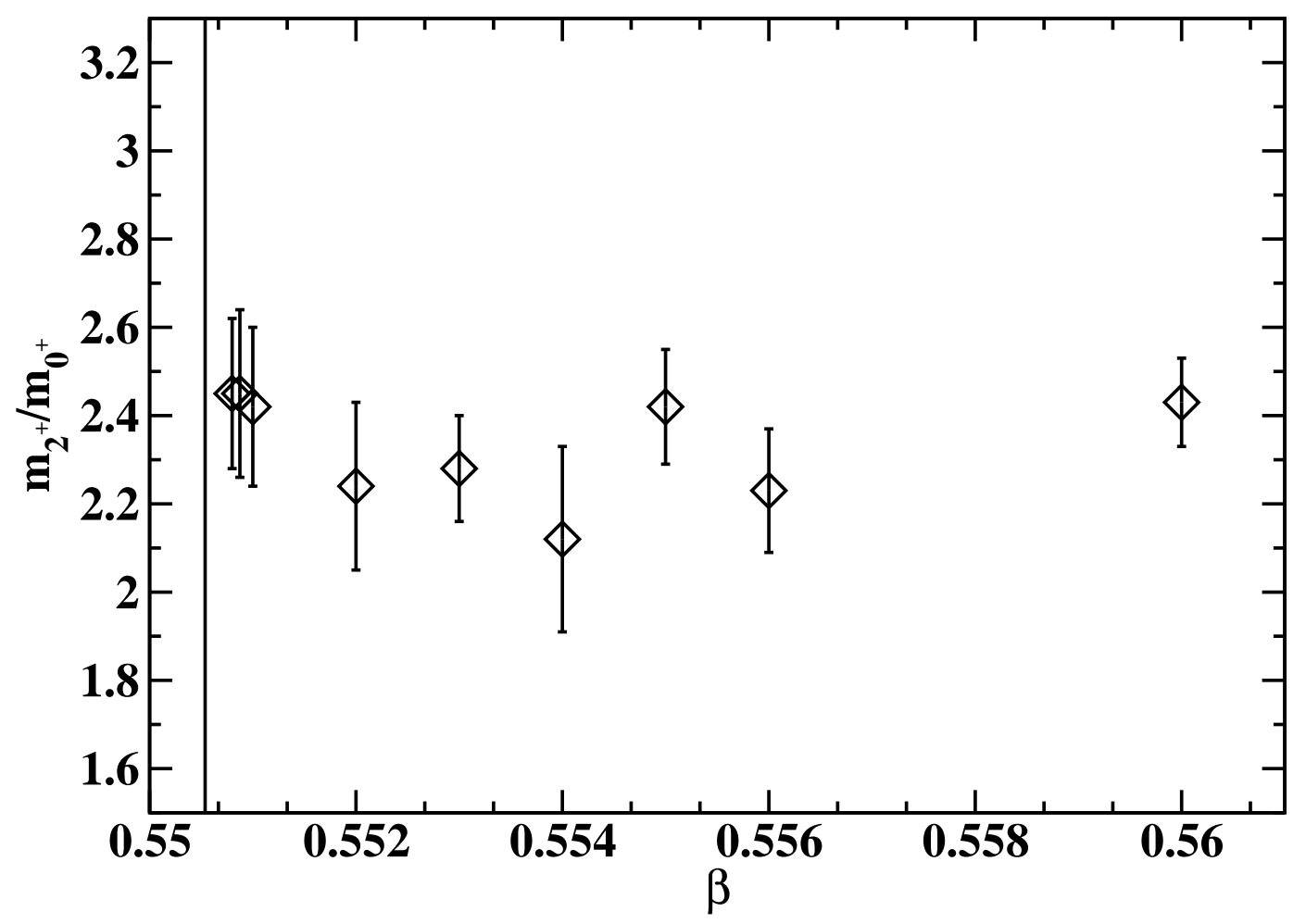

Figure 12: $m_{2^{+}}(\beta) / m_{0^{+}}(\beta)$ for $\beta$ varying in the scaling region. 